\title{
Particle production at HERA
}

Anastasia Grebenyuk for the $\mathrm{H} 1$ and ZEUS collaborations

DESY, Notkestr. 85, D-22607 Hamburg, Germany

\begin{abstract}
Results on particle production in deep-inelastic scattering in ep collision at HERA, obtained with the H1 and ZEUS detectors, are presented. The underlying parton dynamics is investigated by studying the transverse momentum distribution of charged particles and comparing the measurements with various Monte Carlo generators using different approaches to simulate the parton cascade. In this context also results on forward jet production are discussed. The underlying parton dynamics is also studied using prompt photon production in both DIS and photoproduction regimes. The production is compared to theoretical calculations and Monte Carlo predictions. In the context of hadronisation, studies are presented of the scaled momentum distribution for charged hadrons and for $K_{S}^{0}$ and $\Lambda$ particles in the current fragmentation region of the Breit frame. The data are compared to models and to next-to-leading order QCD calculations. In addition, differential cross sections for $K_{S}^{0}$ production in the laboratory and Breit frame are investigated and compared to Monte Carlo predictions, which are used to study the flavour decomposition and strangeness suppression factor. $K_{S}^{0}$ production is also compared to the production of charged particles and to the production rate of the DIS events.
\end{abstract}

Keywords: low x, BFKL dynamics, forward jet, prompt photon, scaled momentum distribution, kaon, strangeness

\section{Parton dynamics}

\subsection{Charged particle transverse momentum spectra}

It has been proposed that in the region of small $x$ at the HERA collider, effects from non- $p_{T}$-ordered parton radiation might become visible. Measurements of charged hadrons in addition to the scattered lepton can be sensitive to the underlying parton dynamics. It was suggested by M. Kuhlen [1] that the transverse momentum spectrum of charged particles is sensitive to whether partons are emitted in a $p_{T}$-ordered cascade (DGLAP) [2] or in an unordered way (beyond DGLAP).

The H1 Collaboration has presented measurements [3] of the transverse momenta and pseudorapidities of charged particles in different regions of $x$ and $Q^{2}$, and compare them to predictions of various Monte Carlo (MC) generators using different approaches to simulate the parton cascade: the RAPGAP generator [4] based on leading-log DGLAP parton showers; the DJANGOH [5] MC which uses the Colour Dipole Model (CDM) [6] as implemented in ARIADNE [7], and which provides a description of gluon emissions which are similar to that of the BFKL evolution [8]; the CASCADE generator [9] based on the CCFM model [10], which unifies the BFKL and DGLAP approaches and requires angular ordering of the emitted partons w.r.t the proton beam. In the CDM and the CCFM approaches the $p_{T}$ of the emitted partons in a parton shower is not ordered. All generators use the Lund string model [11] for hadronisation as implemented in PYTHIA [12]. In addition, H1 uses a fragmentation parameter set tuned by the ALEPH collaboration to fit LEP data [13].

The analysed data set corresponds to an integrated luminosity of $L=88.64 \mathrm{pb}^{-1}$, and the phase space is defined by $5<Q^{2}<100 \mathrm{GeV}^{2}, 0.05<y<0.6,155^{\circ}<$ $\theta_{e}<175^{\circ}$ and $E_{e}>12 \mathrm{GeV}$, with $\theta_{e}$ and $E_{e}$ being the polar angle and energy of the scattered lepton, respectively. Tracks are required to originate from the primary vertex, to lie within the angular range $10^{\circ}<\theta_{l a b}<155^{\circ}$ and to have transverse momenta above $150 \mathrm{MeV}$. The 
data are corrected for detector acceptance, efficiency and resolution effects using Monte Carlo event samples, as well as for the charged decay products of $K_{S}^{0}, \Lambda$ and for other weakly decaying particles. The measured transverse momenta and pseudorapidities of charged particles are presented in the hadronic centre-of-mass system (HCM), i.e. in the rest frame of the virtual photon and proton, and are labeled as $p_{T}^{*}$ and $\eta^{*}$, respectively. In the HCM frame all hadronic final state particles which have $p_{z}^{*}>0$ are said to belong to the current hemisphere, and all particles with $p_{z}^{*}<0$ are assigned to the target or proton remnant hemisphere. All distributions shown are normalised to the total number of DIS events in the analysed phase space.

The $p_{T}^{*}$ spectra of charged particles are presented in Fig. 1 for two pseudorapidity intervals: $1.5<\eta^{*}<4$, where current fragmentation is important, and in the more central region, $0<\eta^{*}<1.5$, where target fragmentation is also playing a role. The DJANGOH (CDM) prediction describes the data fairy well for the whole $p_{T}^{*}$ range, whereas RAPGAP (DGLAP) is below the data for $p_{T}^{*}>1 \mathrm{GeV}$, especially in the central pseudorapidity interval. In contrast, CASCADE (CCFM) is above the data for almost the whole $p_{T}^{*}$ range.

The normalised rapidity distributions are shown in Fig. 2 for $p_{T}^{*}<1 \mathrm{GeV}$ and for $p_{T}^{*}>1 \mathrm{GeV}$ separately. As argued in [1], hadronisation effects should be relevant at small $p_{T}^{*}$, while hard parton radiation should manifest itself in the tail of the $p_{T}^{*}$ distribution. To check the sensitivity to hadronisation effects, RAPGAP predictions with default PYTHIA fragmentation parameters and with parameters tuned by ALEPH are shown in Fig. 2. Significant differences between these two models are seen in the soft $p_{T}^{*}$ region, while for particles with harder transverse momenta this difference is much smaller. Comparing predictions from generators with different QCD scenarios for parton cascades at large $p_{T}^{*}$, where the influence of the fragmentation is much smaller, clear differences between the models with different parton cascades are observed.

The charged particle multiplicity as a function of pseudorapidity in different $x$ and $Q^{2}$ bins are shown in Fig. 3 for $p_{T}^{*}>1 \mathrm{GeV}$. A significant surplus of hard particles in data over the predictions from the DGLAP model RAPGAP is observed at small $x$ and towards the proton remnant. The CASCADE (CCFM) calculations are above the data everywhere, especially at large values of $x$ and $Q^{2}$.

\subsection{Forward jet azimuthal correlations}

To complete the discussion on the sensitivity to parton dynamics, measurements of forward jet production
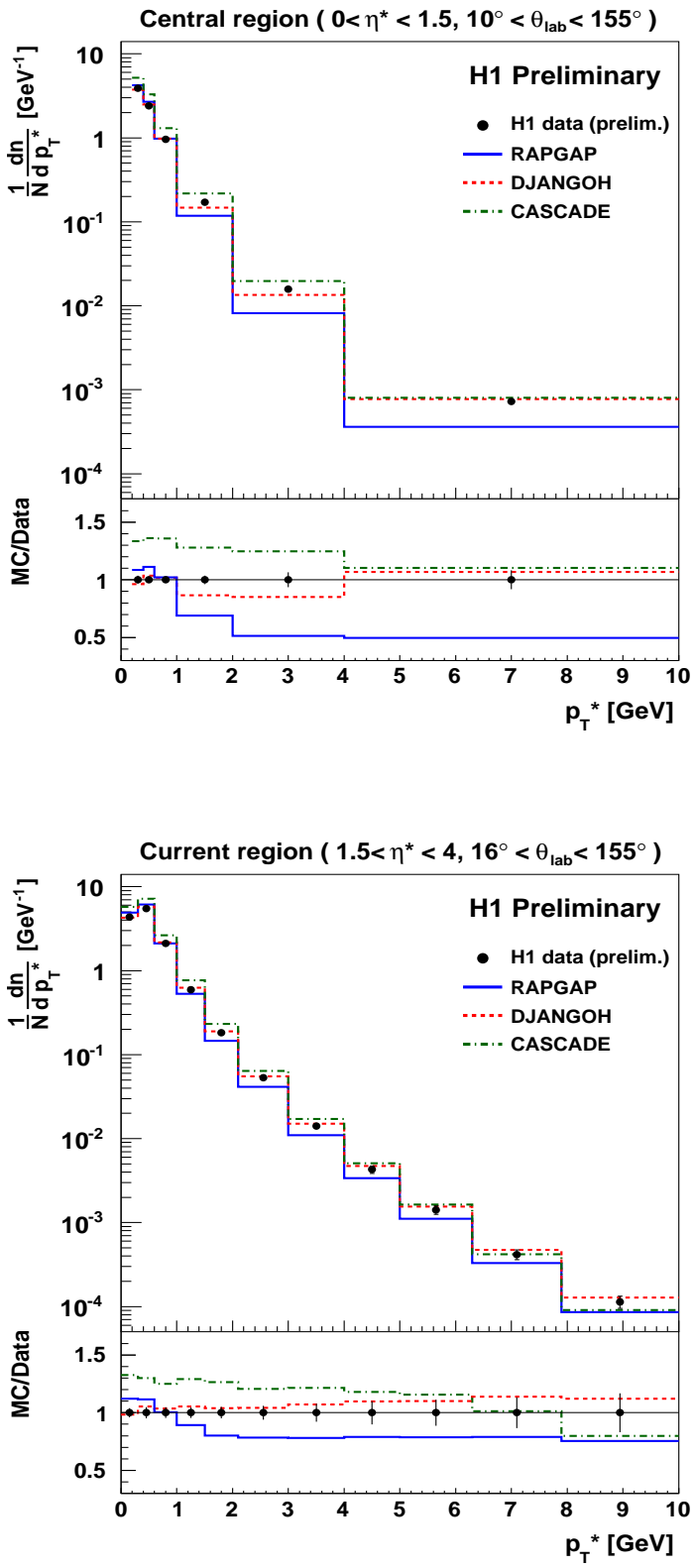

Figure 1: Measured $p_{T}^{*}$ spectra of charged particles in the HCM in the two pseudorapidity intervals: $0.5<\eta^{*}<1.5$ (top) and $1.5<\eta^{*}<4$ (bottom) together with RAPGAP (DGLAP), DJANGOH (CDM) and CASCADE (CCFM) predictions.

$[14,15]$ are presented, that is energetic jets of high transverse momentum produced close to the proton direction in the laboratory frame. Such measurements at low $x$ are considered to be sensitive to BFKL dynamics [16]. Another observables suggested to be sensitive 

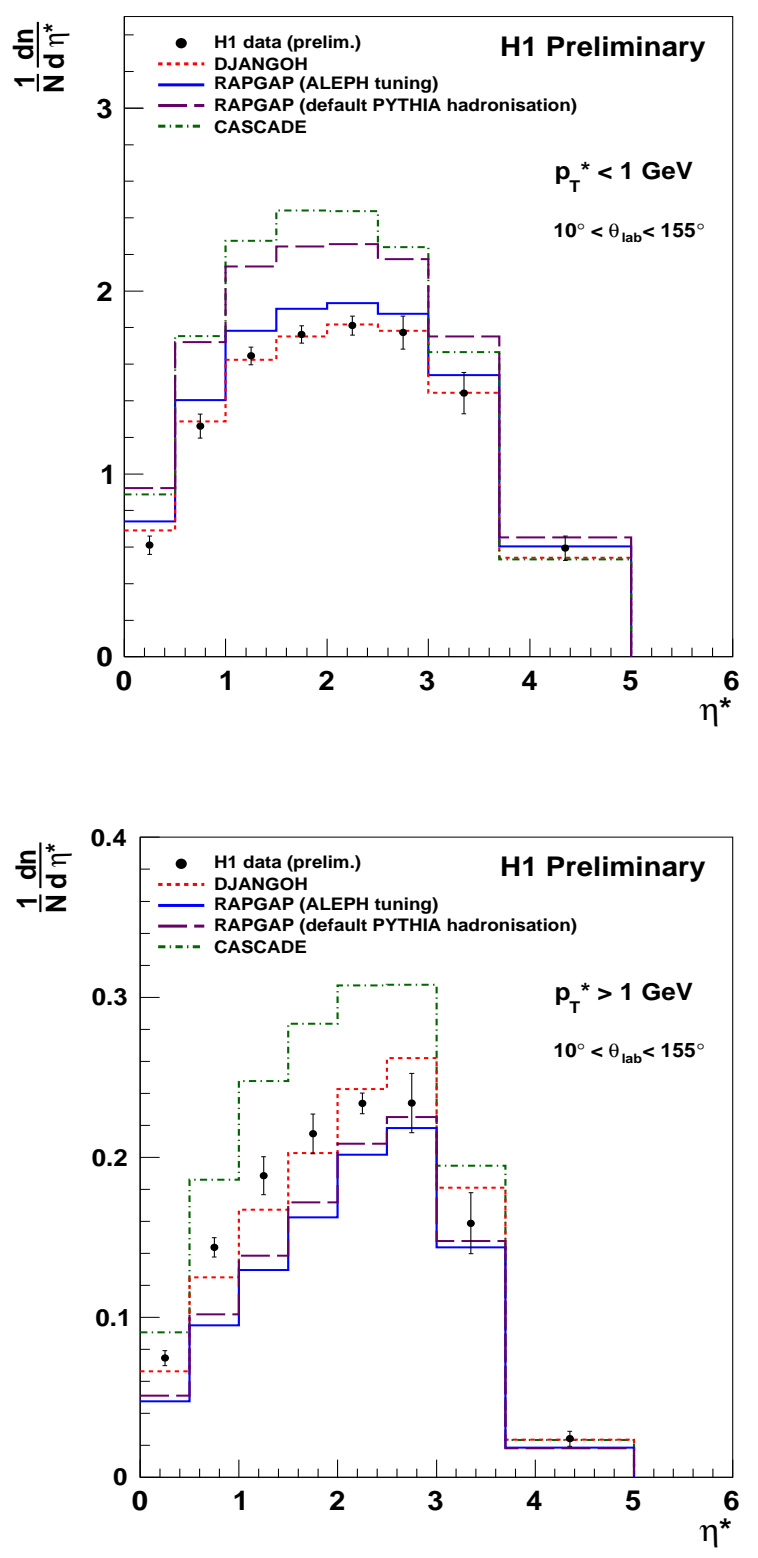

Figure 2: Measured $\eta^{*}$ spectra of charged particles in the HCM for $p_{T}^{*}<1 \mathrm{GeV}$ (top) and for $p_{T}^{*}>1 \mathrm{GeV}$ (bottom) together with RAPGAP (DGLAP), DJANGOH (CDM) and CASCADE (CCFM) predictions. The proton remnant direction is to the left.

to BFKL dynamics [17] is the azimuthal angle difference, $\Delta \phi$, between the forward jet and the scattered lepton, defined in the laboratory frame. In LO $\Delta \phi$ is expected to be close to $\pi$. Inclusion of higher order processes partially decorrelates the jet from the electron.

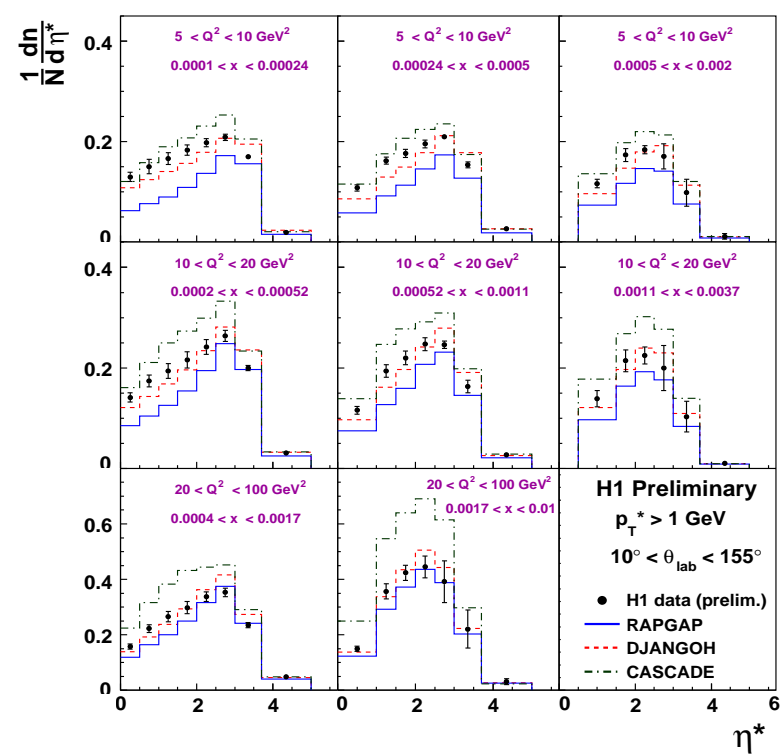

Figure 3: Measured $\eta^{*}$ spectra of charged particles in the HCM for $p_{T}^{*}>1 \mathrm{GeV}$, for eight intervals of $Q^{2}$ and Bjorken $x$ as indicated on the figure, together with Monte Carlo predictions. The proton remnant direction is to the left.

As a consequence, for evolution schemes without ordering in transverse momentum, the decorrelation is expected to increase with the electron-jet rapidity distance, $Y$, since the phase space for additional parton emissions increases. The measurement of such azimuthal correlation is performed by $\mathrm{H} 1$ [18], where the data are compared to predictions of various MC generators using different approaches to simulate the parton cascade, discussed in section 1.1: the RAPGAP generator based on leading-log DGLAP parton showers, the DJANGOH $\mathrm{MC}$ which uses the CDM and the CASCADE generator based on the CCFM model.

The analysed data set corresponds to an integrated luminosity of $L=38.2 \mathrm{pb}^{-1}$, and the phase space is defined by $0.1<y<0.7,5<Q^{2}<85 \mathrm{GeV}^{2}$ and $0.0001<x<0.004$. The analysed sample is required to contain at least one forward jet with transverse momentum and pseudorapidity restricted by $P_{T \text {,fwdjet }}>6$ $\mathrm{GeV}, 1.73<\eta_{\text {fwdjet }}<2.79$, respectively. The forward jets are required to have a fraction of the proton energy, $x_{\mathrm{fwdjet}}=E_{\mathrm{fwdjet}} / E_{p}$ larger than 0.035 to enhance the phase space for BFKL evolution. To suppress the contribution of $k_{T}$-ordered DGLAP cascades the cut $0.5<P_{T \text {,fwdjet }}^{2} / Q^{2}<6$ is applied. If there is more than one jet fulfilling the above requirements, the jet with the largest pseudorapidity is chosen. The data are corrected for detector acceptance, efficiency and resolution effects 
using Monte Carlo event samples.

The cross section $d \sigma / d \Delta \phi$ as a function of $\Delta \phi$ is shown in Fig. 4 for three intervals of the variable $Y=\ln \left(x_{\text {fwdjet }} / x\right): 2.0 \leq Y<3.4,3.4 \leq Y<4.25$ and $4.25 \leq Y<5.75$. These $Y$ bins correspond to average $x$ values of $0.0024,0.0012$ and 0.00048 , respectively. At higher values of $Y$ the forward jet is more

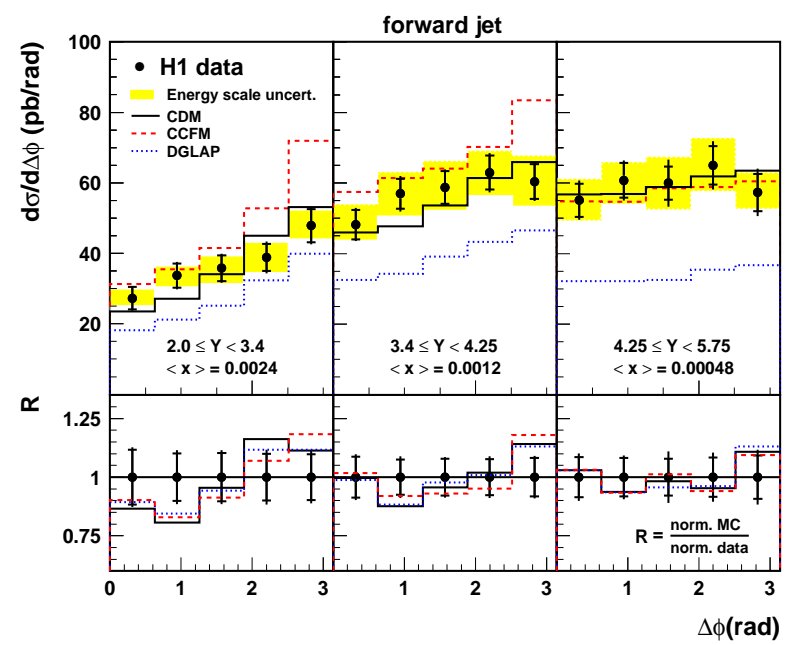

Figure 4: Differential forward jet cross section as a function of the azimuthal angle difference $\Delta \varphi$ between the most forward jet and the scattered lepton in three intervals of the variable $Y=\ln \left(x_{\text {fwdjet }} / x\right)$. The data are compared to predictions of DJANGOH (CDM), CASCADE (CCFM) and RAPGAP (DGLAP). In the lower part of the figure the ratio $R$ of MC to data for normalised cross sections is shown.

decorrelated from the scattered lepton. The cross sections are well described in shape and normalisation by CDM which has a BFKL-like approach. Predictions of RAPGAP (DGLAP) fall below the data, particularly at large $Y$. The prediction of CASCADE (CCFM) overestimates the measured cross section for large $\Delta \phi$ values in the two lowest $Y$ intervals. However, this model provides as good a description as CDM of the data in the highest $Y$ interval. The shape of the $\Delta \phi$ distributions, $1 / \sigma \cdot d \sigma / d \Delta \phi$, is compared to the different MC predictions in the lower part of Fig. 4, where $\sigma$ is the integrated cross section in a given bin of $Y$. The ratio, $R$, is defined as:

$$
R=\left(\frac{1}{\sigma_{M C}} \frac{d \sigma^{M C}}{d \Delta \phi}\right) /\left(\frac{1}{\sigma_{\text {data }}} \frac{d \sigma^{d a t a}}{d \Delta \phi}\right)
$$

The ratio plots show that in the analysed region of phase space the shape of the $\Delta \varphi$ distributions is well described by all MC models. Since the shape predictions of the three models are very similar, this observable alone cannot discriminate among the models.

\subsection{Prompt photons}

Isolated high-energy photons, so-called prompt photons, are a powerful probe of the underlying dynamics, complementary to jets. A prompt photon might be produced in the hard interaction or from the fragmentation of a high momentum quark or gluon in the final state. The latter contribution is suppressed due to the requirement that the outgoing photon is clearly separated from both the outgoing electron and parton. A prompt photon participating in the actual hard subprocess can provide direct information of the process and the proton structure.

In $e p$ collisions isolated high-energy photon production can be studied in both the photoproduction and DIS regimes.

\subsubsection{Prompt photons in photoproduction}

At HERA photoproduction processes arise when the lepton beam emits quasi-real photons which either interact directly with the proton (direct process) or fluctuate into partons which then participate in the hard scattering process (resolved process). In prompt photon production, the direct process is sensitive to the quark content of the proton through the Compton scattering of the exchanged photon with a quark. The resolved process is sensitive to the partonic structure of both the photon and the proton.

In the $\mathrm{H} 1$ analysis presented here [19] the data correspond to an integrated luminosity of $L=340 \mathrm{pb}^{-1}$. Isolated photons with transverse energy $6<E_{T}^{\gamma}<15$ $\mathrm{GeV}$ and pseudorapidity $-1<\eta^{\gamma}<2.4$ are measured in events with the inelasticity $y$ in the range $0.1<y<0.7$. For the photon plus jet subsample, events are selected with a photon candidate and at least one hadronic jet with $-1.3<\eta^{\text {jet }}<2.3$ and $E_{T}^{\text {jet }}>4.5 \mathrm{GeV}$. If more than one hadronic jet is selected, the one with the highest $E_{T}^{\mathrm{jet}}$ is used. The main background is due to photons produced in hadron decays, especially from $\pi_{0}$. For its discrimination from prompt photons, various shower shape variables are used. The data are compared to a QCD calculation based on collinear factorisation in next-to-leading order (FGH) [20] and to a QCD calculation based on the $k_{T}$ factorisation approach (LZ) [21].

Differential inclusive prompt photon cross sections as a function of $E_{T}^{\gamma}$ and $\eta^{\gamma}$ are presented in Fig. 5. The results are compared to QCD calculations labeled FGH and LZ. Both calculations are below the data, most significantly at low $E_{T}^{\gamma}$. The LZ calculation gives a reasonable description of the shape of $\eta^{\gamma}$, whereas the FGH calculation is significantly below the data for central and backward photons $\left(\eta^{\gamma}<0.9\right)$. 

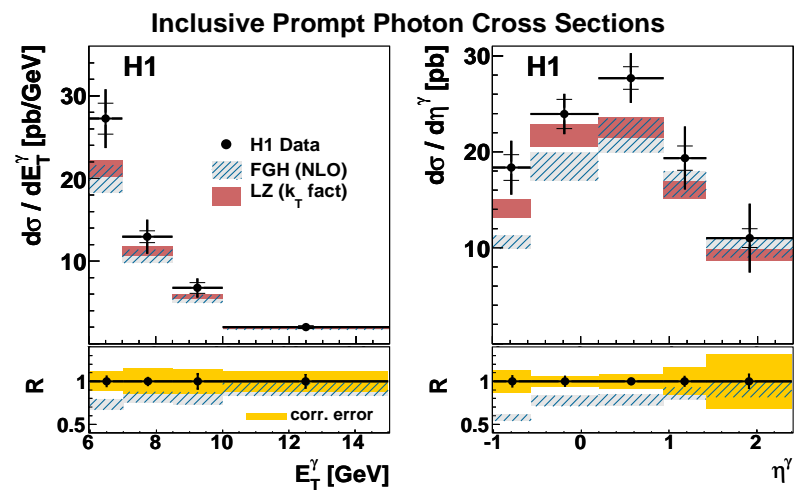

Figure 5: Inclusive differential prompt photon cross sections as a function of prompt photon transverse energy (left) and pseudorapidity (right). The data are compared to a QCD calculation based on collinear factorisation in next-to-leading order (FGH) and to a QCD calculation based on the $k_{T}$ factorisation approach (LZ).

Cross sections for the production of a prompt photon plus jet are presented in Fig. 6 as a function of $\eta^{\gamma}$ and $\eta^{\text {jet }}$. Both calculations show deficits in the description of the $\eta^{\text {jet }}$ shape. Here, the LZ prediction is too high for jets with $\eta^{\text {jet }}<0.5$, and both calculations underestimate the rate of events with forward jets. As in the inclusive case, the FGH prediction is too low for $\eta^{\gamma}<0.2$.
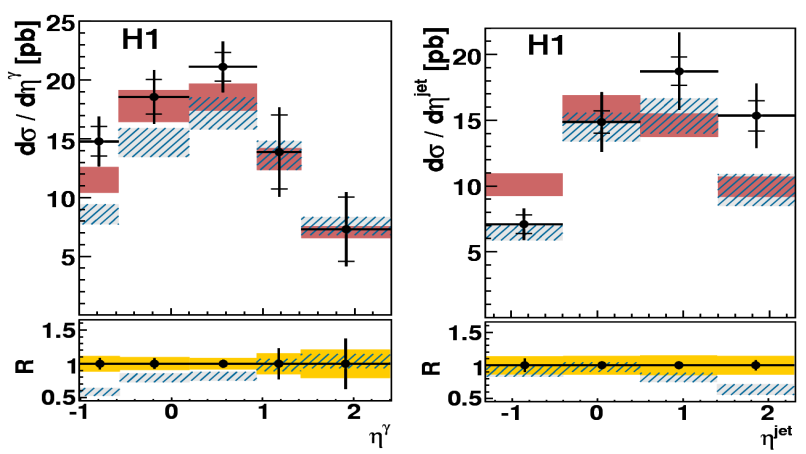

Figure 6: Differential prompt photon plus jet cross sections as a function of $\eta^{\gamma}$ and $\eta^{\text {jet }}$. The data are compared to the QCD calculations shown in Fig. 5.

Another observable, $x^{\mathrm{LO}}$, which is sensitive to the underlying partonic process is :

$$
x_{\gamma}^{\mathrm{LO}}=\frac{E_{T}^{\gamma}\left(e^{-\eta^{\mathrm{jet}}}+e^{-\eta^{\gamma}}\right)}{2 y E_{e}},
$$

where $E_{e}$ is the energy of the scattered lepton. Cross sections for two observables describing the transverse correlation between the photon and the jet, $p_{\perp}$ and $\Delta \phi$, are shown in Fig. 7. In the LO approximation $x_{\gamma}^{\mathrm{LO}}$ corresponds to the longitudinal momentum fraction of the parton in the photon entering the hard interaction. The measurements are shown for $x_{\gamma}^{\mathrm{LO}}>0.8$ where the direct interaction of a photon with the proton dominates. The LZ calculation gives a reasonable description of the $\eta^{\gamma}$. Both calculations show deficits in the description of the $\eta^{\text {jet }}$ shape and underestimate the tails of the $\Delta \phi$ and $p_{\perp}$ distributions.
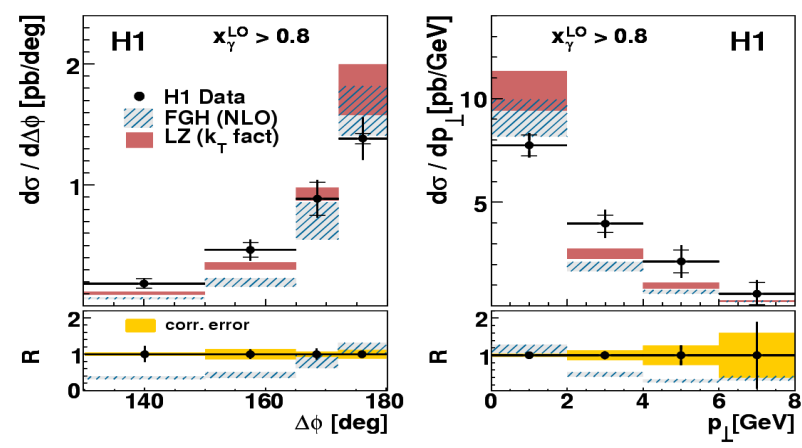

Figure 7: Differential prompt photon plus jet cross sections as a function of the photon momentum transverse to the jet direction, $p_{\perp}$, and the difference in azimuthal angle between the photon and the jet, $\Delta \phi$. The data are compared to the QCD calculations FGH and LZ.

\subsubsection{Prompt photons in DIS}

The cross sections for isolated photons in DIS have been calculated to order $\alpha_{s}^{3}$ (GGP) [22]. According to the GGP approach, three processes contribute to the $e p \rightarrow e \gamma X$ cross section: one contribution comes from the radiation of a photon from the quark line (QQ photon), the second comes from the radiation of the photon from the lepton line (called LL photon), and the third contribution which is due to photons from jet fragmentation (LQ photon) is suppressed. The cross sections [23] measured by ZEUS are compared to leadingorder MC models, which use the QCD parton shower approach to incorporate higher-order QCD effects followed by fragmentation into hadrons. DIS events with QQ photon emission were generated by the MC generator PYTHIA and LL photons were simulated using ARIADNE.

The analysed data set corresponds to an integrated luminosity of $L=332 \mathrm{pb}^{-1}$, and the phase space is defined by $10<Q^{2}<350 \mathrm{GeV}^{2}, 140^{\circ}<\theta_{e}<180^{\circ}$ and $E_{e}>10 \mathrm{GeV}$, with $\theta_{e}$ and $E_{e}$ being the polar angle and energy of the scattered lepton, respectively. Isolated photons with transverse energy $4<E_{T}^{\gamma}<15 \mathrm{GeV}$ and pseudorapidity $-0.7<\eta^{\gamma}<0.9$ are measured. The DIS events are selected with a photon candidate and at least one hadronic jet with $-1.5<\eta^{\text {jet }}<1.8$ 
and $E_{T}^{\mathrm{jet}}>2.5 \mathrm{GeV}$. If more than one hadronic jet is selected, the one with the highest $E_{T}^{\mathrm{jet}}$ is used.

Differential cross sections for prompt photon production associated with jets are presented as a function of $E_{T}^{\gamma}, E_{T}^{\mathrm{jet}}, Q^{2}$ and $x$. The cross sections are compared in Figs. 8 and 9 to the sum of the QQ predictions from PYTHIA (scaled by a factor 1.6) and the LL predictions from ARIADNE. The sum of the two MC predictions
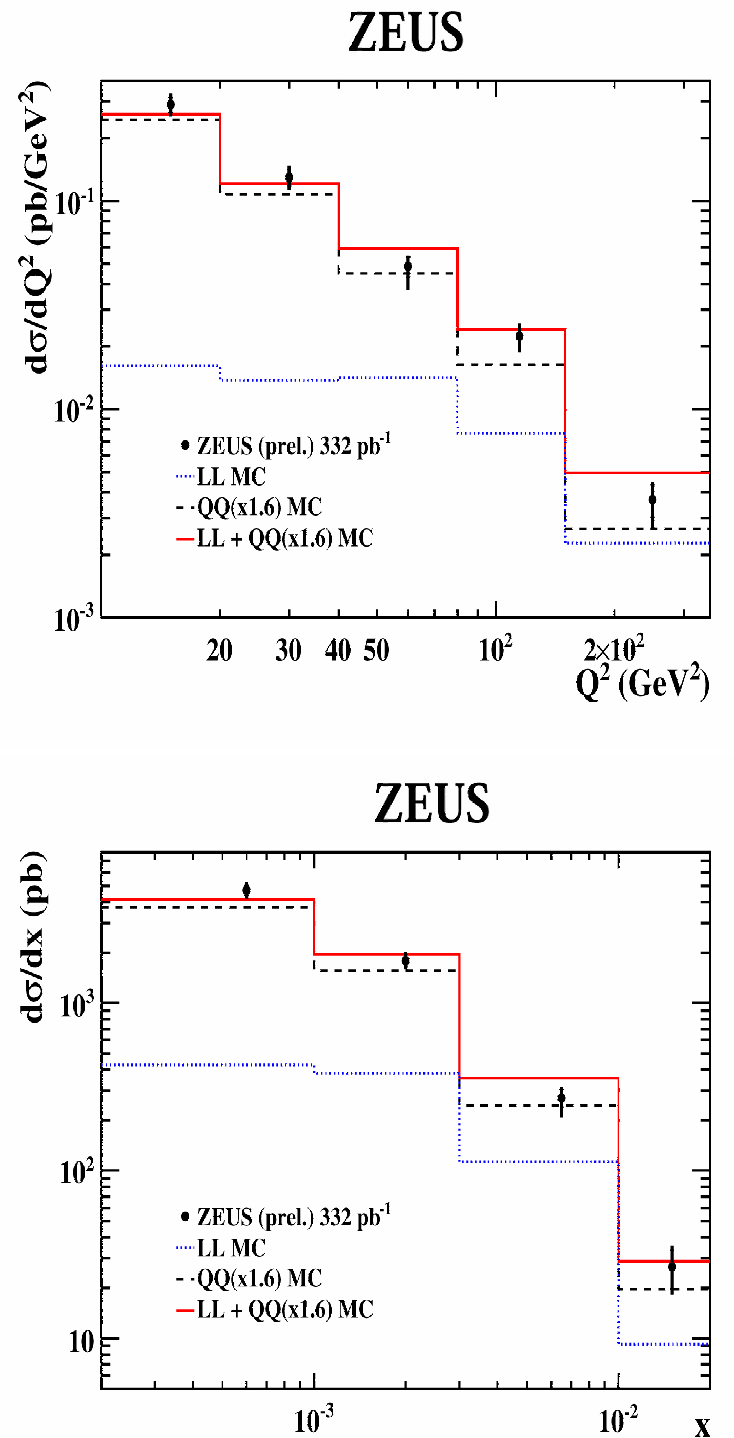

Figure 8: Differential prompt photon plus jet cross sections as a function of $Q^{2}$ (top) and $x$ (bottom). The solid histograms are the MC predictions from the sum of QQ photons from PYTHIA scaled by a factor 1.6 plus LL photons from DJANGOH. The dashed (dotted) lines show the QQ (LL) contributions.
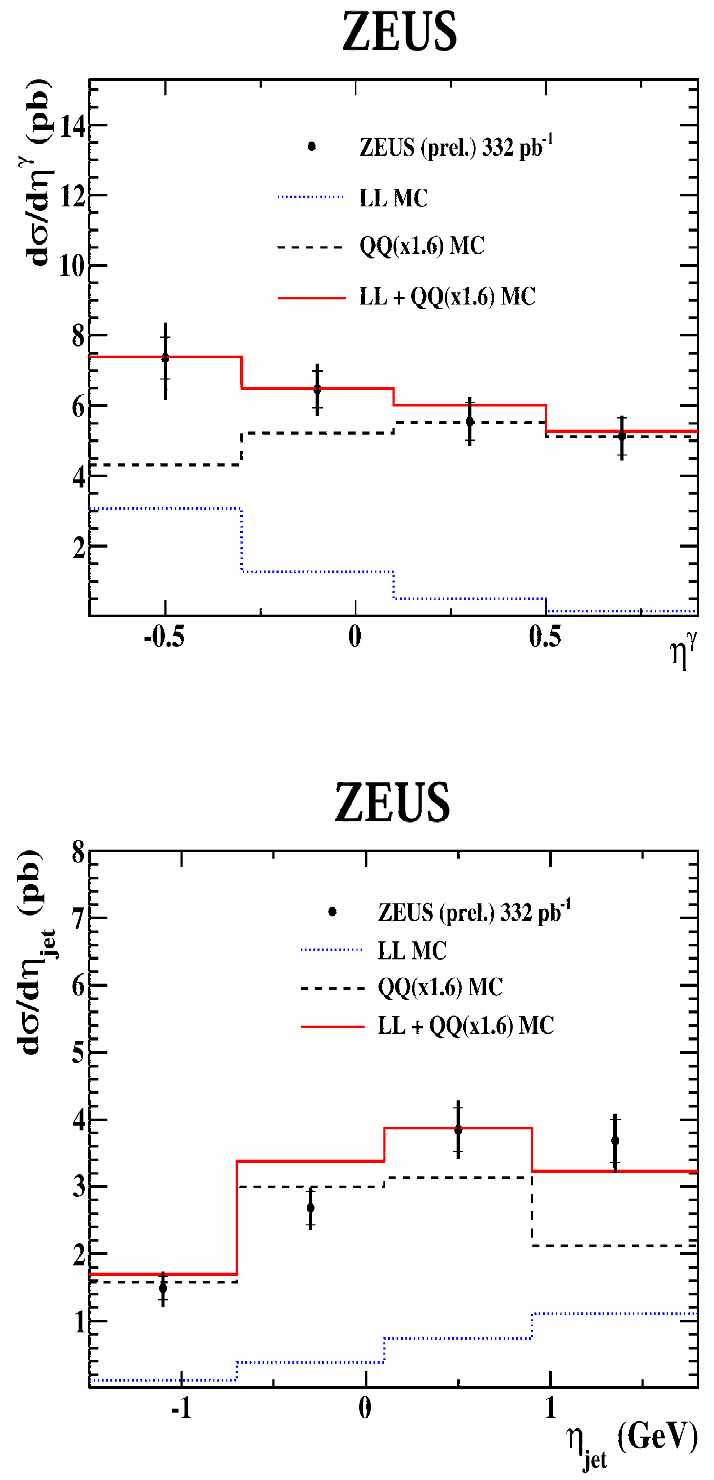

Figure 9: Differential prompt photon plus jet cross sections as a function of $\eta^{\gamma}$ (top) and $\eta^{\text {jet }}$ (bottom). The solid histograms are the MC predictions from the sum of QQ photons from PYTHIA scaled by a factor 1.6 plus LL photons from DJANGOH. The dashed (dotted) lines show the QQ (LL) contributions.

gives a good description of the data.

\section{Hadronisation}

\subsection{Scaled momentum distributions}

An investigation of the production of charged particles as well as $K_{S}^{0}$ mesons and $\Lambda$ baryons in the current fragmentation region of the Breit frame is presented. 
Multiplicity distributions are measured as a function of $Q^{2}$ per unit of the scaled momentum, $x_{p}=2 p^{\text {Breit }} / Q$. Here, $p^{\text {Breit }}$ denotes the momentum of a hadron in the Breit frame. The aim was to check the universality of the quark fragmentation function and the factorisation theorem approach used to predict hadron production in different processes. The data presented in this section are corrected for detector acceptance, efficiency and resolution effects. In the case of inclusive scaled momentum distributions of charged particles, the data are corrected for the charged decay products of $K_{S}^{0}, \Lambda$ and for other weakly decaying particles. All distributions shown are normalised to the total number of DIS events, $N$, in the analysed phase space.

\subsubsection{Scaled momentum distributions of charged particles}

To check the universality of the fragmentation functions the measured scaled momentum distributions of charged particles from ep collision [24, 25, 26] at HERA and from $e^{+} e^{-}$experiments [27] are compared. The particle momenta in a hemisphere of the $e^{+} e^{-}$annihilation are scaled to half of the centre-of-mass energy, $\sqrt{s} / 2$. The results are shown shown in Fig. 10. General agreement is observed between $e p$ and $e^{+} e^{-}$collisions which supports the concept of quark-fragmentation universality. The scaling violations can be seen for charged particles for large $Q^{2}$ values: with increasing $Q^{2}$ the phase space for soft gluon radiation gets enlarged, which leads to a rise in the number of soft particles at small $x_{p}$ and to a decrease in the number of those with high $x_{p}$.

The data obtained by H1, shown in Fig. 10, were further investigated to study the asymmetry in the number of positively and negatively charged particles [28]. Hadrons with small values of $x_{p}$ are predominately produced by fragmentation, while hadrons at large $x_{p}$ are more likely to contain a parton from the hard interaction. Therefore a study of the $x_{p}$ distribution separately for positively and negatively charged particles should reveal information about the valence quarks and their fragmentation. The data used for these studies correspond to an integrated luminosity of $44 \mathrm{pb}^{-1}$, and the phase space is defined by $100<Q^{2}<8000 \mathrm{GeV}^{2}$ and $0.05<y<0.6$.

Figure 11 shows the scaled momentum distribution $\left(D\left(x_{p}, Q\right)=\frac{1}{N} \frac{d n}{d x_{p}}\right)$ as a function of $Q$ in different $x_{p}$ intervals. The charge asymmetry observed at large $x_{p}$ evolves to larger values as $Q$ increases. The largest asymmetries are obtained in the highest $Q$ and highest $x_{p}$ intervals. It should be noted that higher average $Q$ corresponds to higher average Bjorken $x$ and hence
ZEUS
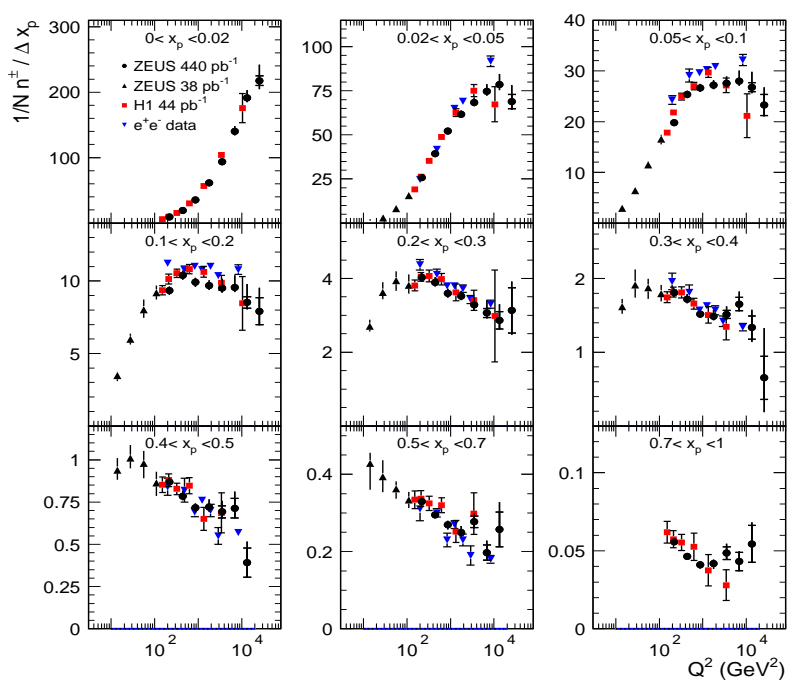

Figure 10: Number of charged particles per event per unit of $x_{p}$, $1 / N n^{ \pm} / \Delta x_{p}$, as a function of $Q^{2}$ in $x_{p}$ bins with width $\Delta x_{p}$.
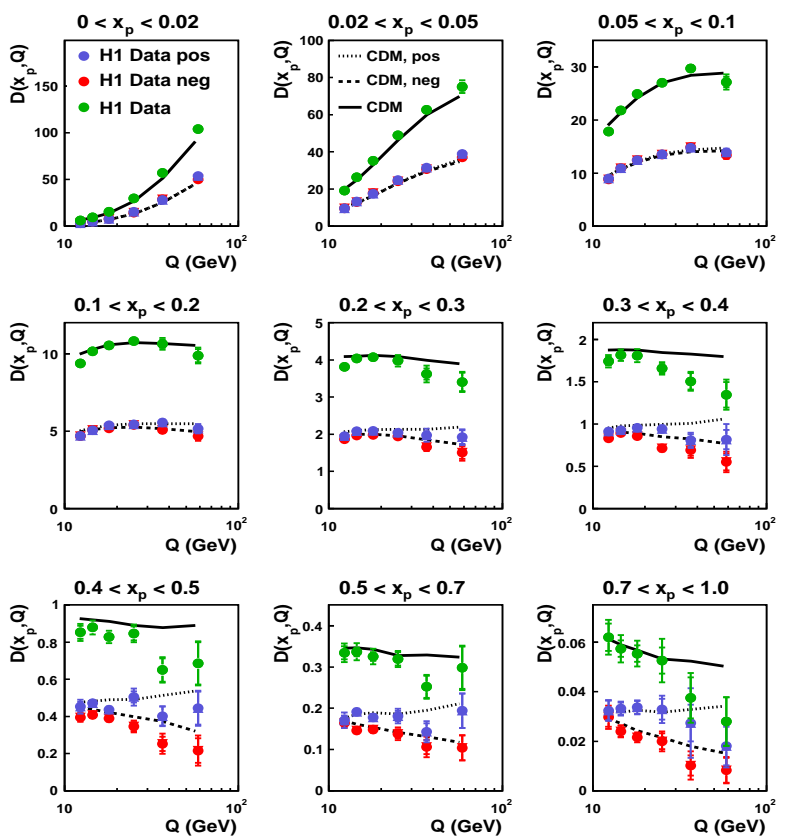

Figure 11: Normalised distributions of the scaled momentum, $D\left(x_{p}, Q\right)$, for all charged particles and for positively (pos), and negatively (neg), charged particles separately, as a function of $Q$ for nine different $x_{p}$ regions. The data are compared to predictions from the DJANGOH (CDM) Monte Carlo program.

the highest $Q$ intervals are most sensitive to the valence quark distribution. The scaled momentum distributions are roughly predicted by the DJANGOH predic- 
tions based on the CDM.

\subsubsection{Comparison to NLO QCD calculations}

The scaled momentum distributions were also compared to next-to-leading order QCD calculations [29]. Various fragmentation functions (FFs), obtained from fits to $e^{+} e^{-}$data $[30,31]$ (Kretzer, KKP), to $e^{+} e^{-}$and $p p / p \bar{p}$ data [32] (AKK), and to $e^{+} e^{-}, p p / p \bar{p}$ and $e p$ data [33] (FSS), were used in the next-to-leading order (NLO) QCD calculations.

The scaled momentum distributions obtained by ZEUS, shown in Fig. 12, are compared now to various NLO QCD calculations. The kinematic range of the high luminosity sample $\left(L=440 \mathrm{pb}^{-1}\right)$ corresponds to $160<Q^{2}<40960 \mathrm{GeV}^{2}$ and $0.002<x<0.75$ [25]. The previously published results [26] cover the lower $Q^{2}$ range of $10<Q^{2}<160 \mathrm{GeV}^{2}$ and correspond to an integrated luminosity of $L=38 \mathrm{pb}^{-1}$. The uncertainties

\section{ZEUS}
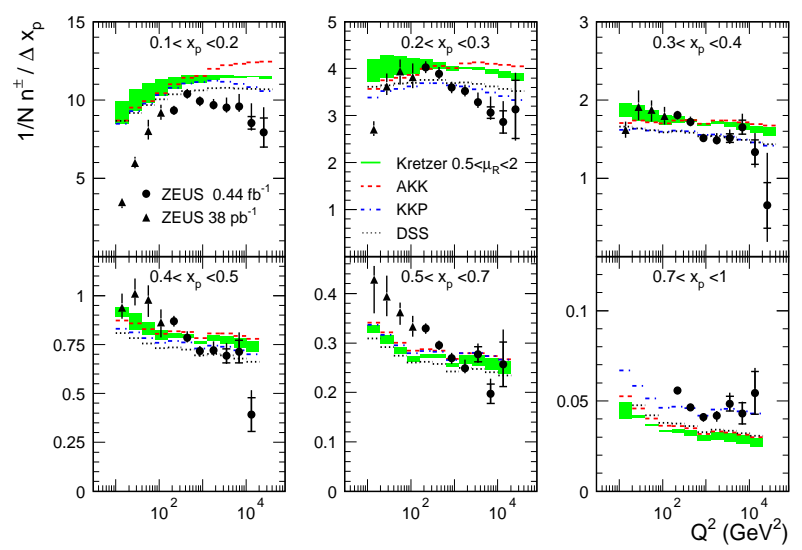

Figure 12: Scaled momentum distributions as a function of $Q^{2}$ in different regions of $x_{p}$ for charged particles together with predictions of NLO QCD using different fragmentation functions.

are illustrated for the calculation of Kretzer only. The NLO calculations do not provide a good description of the data. They show a different $x_{p}$ slope, and the scaling violations predicted are not strong enough.

\subsection{3. $K_{S}^{0}$ and $\Lambda$ scaled momentum distributions}

Scaled momentum distributions for $K_{S}^{0}$ mesons and $\Lambda$ baryons were measured by ZEUS [34] in the kinematic range $10<Q^{2}<40000 \mathrm{GeV}^{2}$ and $0.001<x<0.75$. The data correspond to an integrated luminosity of $L=$ $290 \mathrm{pb}^{-1}$.

Figures 13 and 14 show the scaled momentum spectra, as a function of $Q^{2}$ in different regions of $x_{p}$, for $K_{S}^{0}$ and $\Lambda$ production, respectively. The NLO QCD predictions using two different FFs describe the data only in certain regions of the phase space. For the $\mathrm{AKK}+\mathrm{CYCLOPS}$ calculation this is limited to $0.6<x_{p}<1$, whereas the DSS calculation describes the $K_{S}^{0}$ data adequately, except for regions of low $x_{p}$ and $Q^{2}$. Together with the NLO QCD calculations the predictions of MC generators ARIADNE based on CDM and LEPTO [35] based on leading-log DGLAP parton shower are shown in Figs. 13 and 14. Both predictions give a reasonable description of the data over almost the whole phase space.

\section{ZEUS}
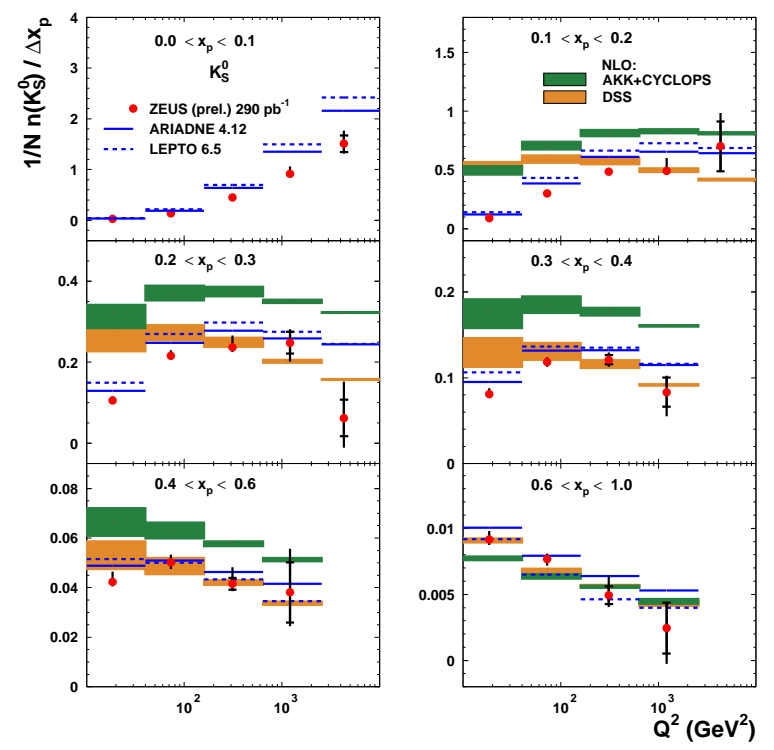

Figure 13: Scaled momentum distributions as a function of $Q^{2}$ in different regions of $x_{p}$ for $K_{S}^{0}$ together with predictions of NLO QCD and LO QCD models implemented in Monte Carlo programs.

\subsection{Strangeness production}

The production of strange hadrons in high energy particle collisions allows the investigation of strong interactions in the perturbative and non-perturbative regimes. Strange quarks are created in the non-perturbative process of colour string fragmentation, which constitutes the dominant production mechanism of strange hadrons. Measurements of strangeness production have been used by $\mathrm{H} 1$ to investigate the suppression of strangeness relative to lighter flavours in fragmentation, as given by the strangeness supression factor $\lambda_{s}$ in the Lund fragmentation model. 


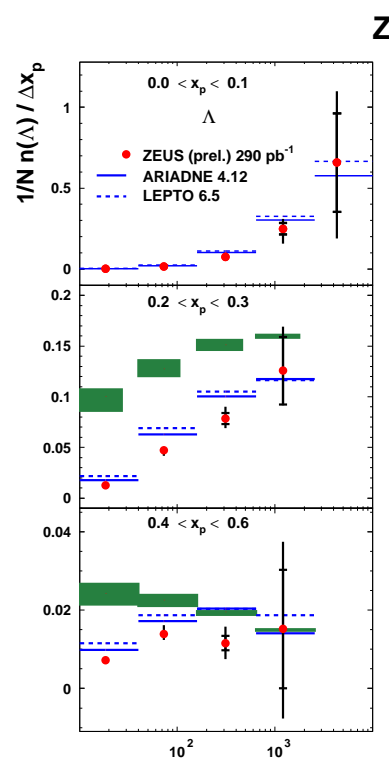

ZEUS

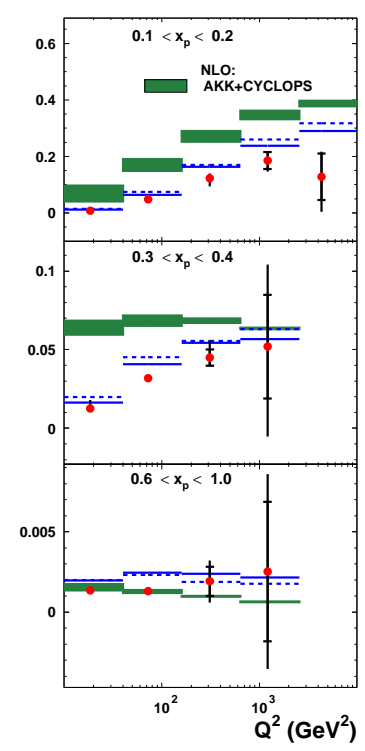

Figure 14: Scaled momentum distributions as a function of $Q^{2}$ in different regions of $x_{p}$ for $\Lambda$ baryons together with predictions of NLO QCD and LO QCD models implemented in Monte Carlo programs.

In the present analysis [36] the $K_{S}^{0}$ meson is studied through its decay channel $K_{S}^{0} \rightarrow \pi^{+} \pi^{-}$. The differential cross section as function of the transverse momentum $p_{T}$ of the strange particle is determined in the laboratory frame. The analysis is also carried out in the Breit frame as function of the scaled momentum fraction $x_{p}^{\mathrm{BF}}$ and the transverse momentum $p_{T}^{\mathrm{BF}}$ in both, current $(\mathrm{CBF})$ and target (TBF) hemispheres. Two models are used for the comparison to data, ARIADNE based on CDM and LEPTO based on DGLAP evolution. The data correspond to an integrated luminosity of $L=340 \mathrm{pb}^{-1}$. The analysis phase space is defined by $145<Q^{2}<20000$ $\mathrm{GeV}^{2}$ and $0.2<y<0.6$.

Figure 15 shows the flavour decomposition of $K_{S}^{0}$ production as a function of $p_{T}$ as obtained by the LEPTO Monte Carlo program. It indicates that $K_{S}^{0}$ mesons originate mainly from the fragmentation of $u$ and $d$ quarks in this analysis. The second largest contribution is obtained from the decay of heavy flavoured hadrons. Strange quarks contribute only very little to the $K_{S}^{0}$ production cross section in general.

Figure 16 shows the differential cross section of $K_{S}^{0}$ production as a function of $p_{T}$ in the laboratory frame and of the scaled momenta $x_{p}^{\mathrm{CBF}}$ and $x_{p}^{\mathrm{TBF}}$ in the current and target hemisphere of the Breit frame, respectively. The measurements are compared to the CDM

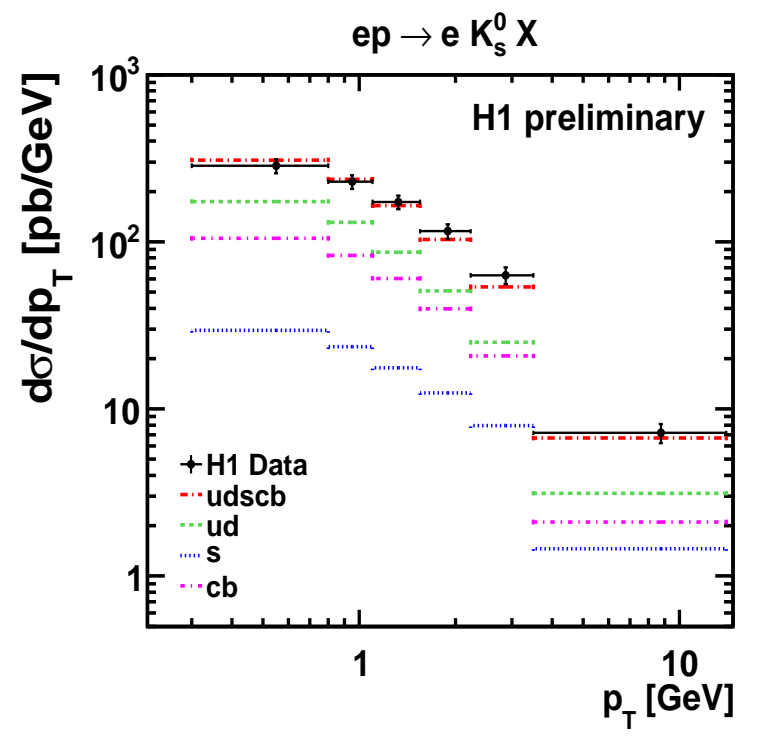

Figure 15: Differential cross sections for $K_{S}^{0}$ separated by flavour contributions as function of the transverse momentum $p_{T}$ in the laboratory frame.

and MEPS models with two different values of the strangeness suppression parameter, $\lambda_{s}=0.22$ and $\lambda_{s}=$ 0.286. The two models with $\lambda_{s}=0.286$ give similar descriptions and agree better with the data in shape and normalisation than do the models with $\lambda_{s}=0.22$. The $\mathrm{CBF}$ is mainly sensitive to the dynamics of the hard subprocess while in the TBF the influence from hadronization is expected to be significant. Therefore, more sensitivity to $\lambda_{s}$ is expected in $x_{p}^{\mathrm{TBF}}$ compared to $x_{p}^{\mathrm{CBF}}$. However, the data of the current measurements are not precise enough to see significant differences in the sensitivity to hadronization of the two hemispheres.

The differential ratio of $K_{S}^{0}$ to charged particle production in the same phase space is shown in Fig. 17 as function of $Q^{2}$ (top) and $p_{T}$ (bottom) in the laboratory frame. This ratio is almost constant in $Q^{2}$ while it rises significantly with $p_{T}$, which is expected due to the heavier mass of the $K_{S}^{0}$ relative to $\pi^{ \pm}$the most common charged particles. All distributions agree best with the predictions for a strangeness suppression factor $\lambda_{s}=0.286$.

The production rate of $K_{S}^{0}$ per DIS event, selected in the same kinematic phase space, has a flat distribution around 0.4 independently of $Q^{2}$ as shown in Fig. 18 . Again, both models with $\lambda_{s}=0.286$ describe the data well. 

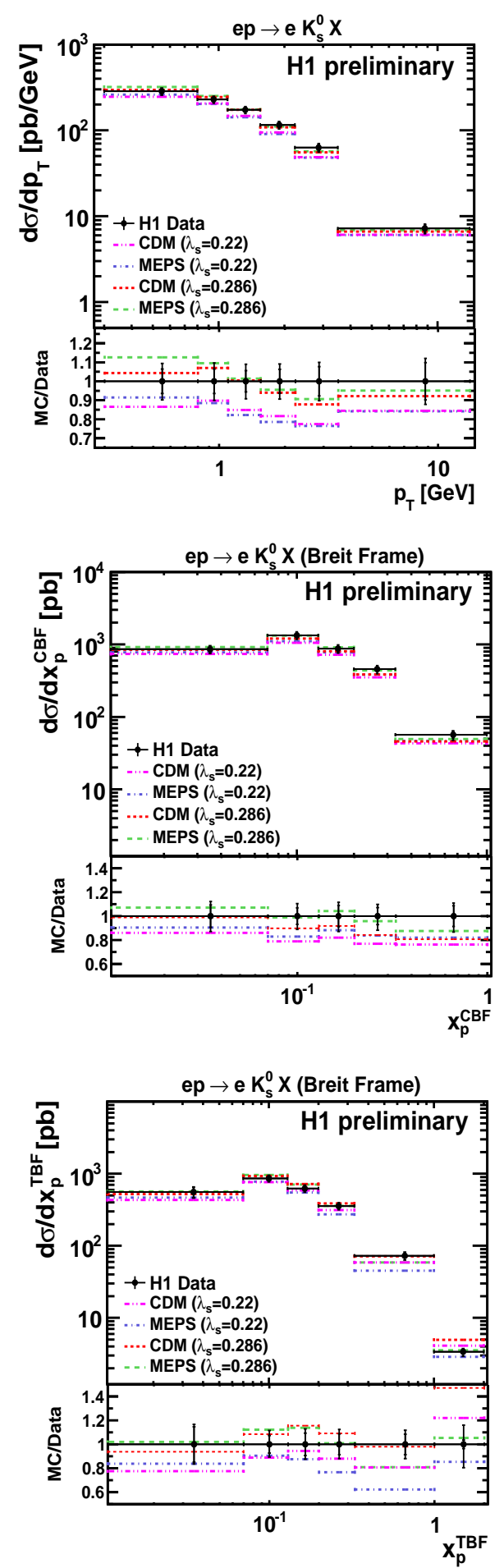

Figure 16: Differential cross sections for $K_{S}^{0}$ as function of the transverse momentum in the laboratory frame (top), and the scaled momentum fraction in the current $x_{p}^{\mathrm{CBF}}$ (middle) and target $x_{p}^{\mathrm{TBF}}$ (bottom) hemispheres in the Breit frame.
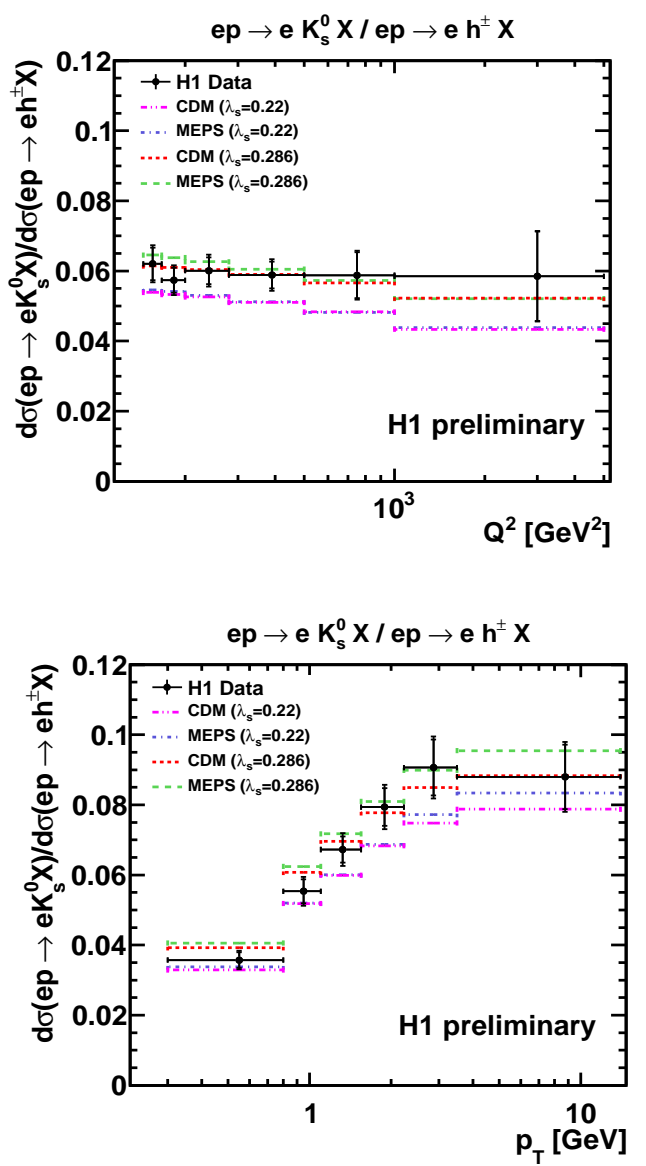

Figure 17: Ratios of $K_{S}^{0}$ over charged particle production as function of $Q^{2}$ (top) and transverse momentum $p_{T}$ (bottom).

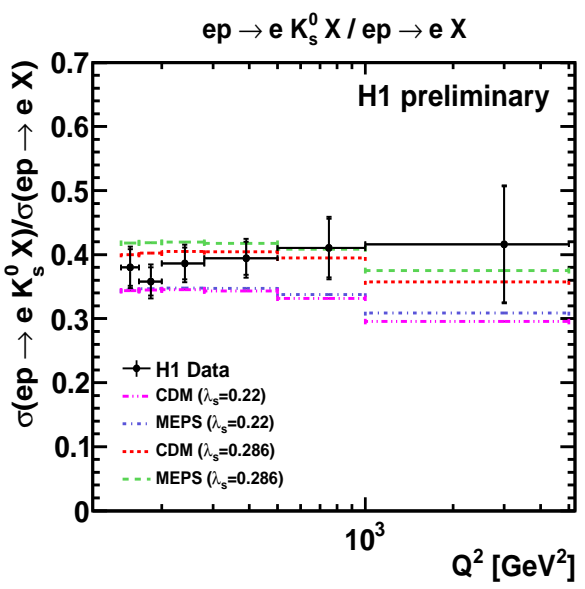

Figure 18: Rate of $K_{S}^{0}$ production as function of $Q^{2}$. 


\section{Conclusion}

The production of charged particles has been studied in deep-inelastic scattering at HERA. The investigation of the underlying parton dynamics by measuring the transverse momentum of charged particles, and forward jet production shows the importance of parton emissions unordered in transverse momentum. A QCD model, exhibiting this feature, such as the BFKL-like colour dipole model is best in the description of the data, whereas a model generating emissions according to the DGLAP approach undershoots the data at low Bjorken $x$. It is also shown that hadronisation effects are important at low $p_{T}^{*}$ (in the hadronic centre-of-mass system) of charged particles, while differences in hard parton radiation of the models manifest themselves in the tail of the transverse momentum distribution.

Results on prompt photons are presented in photoproduction and DIS regimes. In photoproduction the inclusive prompt photon cross sections and cross sections for photon plus jet are compared to a QCD calculations based on the collinear factorisation in NLO and on the $k_{T}$ factorisation approach. Calculations generally underestimate cross sections and fail to describe shapes in several kinematical regions. In the DIS regime, the cross sections for photon plus jet are compared to a Monte Carlo model. Good agreement is observed after scaling the predictions by a constant.

In the context of hadronisation studies scaled momentum distribution for charged hadrons as well as for $K_{S}^{0}$ and $\Lambda$ particles in the current fragmentation region of the Breit frame are presented. The measurements show scaling violations. Next-to-leading order QCD calculations based on different fragmentation functions can describe the data only in some regions of $x_{p}$. In addition, the scaled momentum distribution is used to study the charge asymmetry. It is found to depend on the scaled momentum $x_{p}$, indicating a larger asymmetry for large $x_{p}$. The observed charge asymmetry is reproduced by the various models.

Strangeness production is investigated by looking at the $K_{S}^{0}$ cross sections as a function of $p_{T}$ in the laboratory frame and of the scaled momenta $x_{p}^{\mathrm{CBF}}$ and $x_{p}^{\mathrm{TBF}}$ in the current and target hemisphere of the Breit frame. It is shown that $K_{S}^{0}$ production at HERA is dominated by fragmentation in the kinematic range of this analysis. All measurements are found to be described best by a strangeness suppression factor $\lambda_{s}=0.286$ independently of the models under consideration.

\section{Bibliography}

\section{References}

[1] M. Kuhlen, Phys. Lett. B 382 (1996) 441.

[2] V. Gribov and V. Lipatov, Sov. J. Nucl. Phys. 15 (1972) and 675; V. Lipatov, Sov. J. Nucl. Phys. 20 (1975) 94; G. Altarelli and G. Parisi, Nucl. Phys. B 126 (1977) 298; Y. Dokshitzer, Sov. Phys. JETP 46 (1977) 641.

[3] H1 Preliminary DIS2011, H1 prelim-11-035.

[4] H. Jung, Comp. Phys. Commun. 86 (1995) 147.

[5] K. Charchula, G.A. Schuler and H. Spiesberger, Comp. Phys. Commun. 81 (1994) 381.

[6] B. Andersson et al., Z. Phys. C 43 (1989) 625; L. Lönnblad, Comput. Phys. Commun. 71 (1992) 15.

[7] L. Lönnblad, ARIADNE 4.10, Comput. Phys. Commun. 71 (1992) 15.

[8] E. Kuraev, V. Lipatov and V. Fadin, Sov. Phys. JETP 44 (1976) 443, Sov. Phys. JETP 45 (1977) 199; Y. Balitsky and V. Lipatov, Sov. J. Nucl. Phys. 28 (1978) 822.

[9] H. Jung, Comp. Phys. Commun. 143 (2002) 100.

[10] M. Ciafaloni, Nucl. Phys. 296 (1988) 49; M. Catani, F. Fiorani and G. Marchesini, Phys. Lett. 234 (1990) 339, Nucl. Phys. 336 (1990) 18.

[11] B. Andersson, G. Gustafson, G. Ingelman and T. Sjostrand, Phys. Rept. 97 (1983) 31.

[12] T. Sjostrand et al., PYTHIA V6.1, Comp. Phys. Commun. 135 (2001) 238.

[13] S. Schael et al. [ALEPH Collaboration], "Bose-Einstein correlations in W-pair decays with an event-mixing technique" Phys. Lett. B 606 (2005) 265; G. Rudolph [ALEPH Collaboration], private communication.

[14] A. Aktas et al. [H1 Collaboration], Eur. Phys. J. C 46 (2006) 27 [arXiv:hep-ex/0508055].

[15] S. Chekanov et al. [ZEUS Collaboration], Eur. Phys. J. C 52 (2007) 515 [arXiv:hep-ex/0707.3093].

[16] A.H. Mueller, Nucl. Phys. B (Proc. Suppl.) 18 C (1990) 125.

[17] J. Bartels et al., Phys. Lett. 384 (1996) 300 [arXiv:hep$\mathrm{ph} / 9604272]$

[18] F.D. Aaron et al. [H1 Collaboration], submitted to EPJC [arXiv:hep-ex/1111.4227].

[19] F.D. Aaron et al. [H1 Collaboration], Eur. Phys. J. C 66 (2010) 17.

[20] M. Fontannaz, J.P. Guillet and G. Heinrich, Eur. Phys. J. C 21 (2001) 303 [arXiv:hep-ph/0105121];

M. Fontannaz and G. Heinrich, Eur. Phys. J. C 34 (2004) 191 [arXiv:hep-ph/0312009]

[21] A.V. Lipatov and N.P. Zotov, Phys. Rev. D 72 (2005) 054002 [hep-ph/0506044].

[22] G. Kramer, A. Gehrmann-De Ridder and H. Spiesberger., Nucl. Phys. B 578 (2000) 326;

A. Gehrmann-De Ridder, T. Gehrmann and E. Poulsen., Phys. Rev. Lett. 96 (2006) 132002, Eur. Phys. J. C 47 (2006) 395.

[23] ZEUS Preliminary DIS2011, ZEUS-prel-11-007.

[24] F.D. Aaron et al. [H1 Collaboration], Phys. Lett. B 654 (2007) 148.

[25] H. Abramowicz et al. [ZEUS Collaboration], JHEP 6 (2010) 009.

[26] J. Breitweg et al. [ZEUS Collaboration], Eur. Phys. J. C 11 (1999) 251

[27] A. Petersen et al. [MARK II Collaboration], Phys. Rev. D 37 (1988) 1;

W. Braunschweig et al. [TASSO Collaboration], Z. Phys. C 47 (1990) 187;

Y.K. Li et al. [AMY Collaboration], Phys. Rev. D 41 (1990) 
2675

P. Abreu et al. [DELPHI Collaboration], Phys. Lett. B 311 (1993) 408.

[28] F. D. Aaron et al. [H1 Collaboration], Phys. Lett. B 681 (2009) 125.

[29] D. Graudenz, Phys. Rev. B 406 (1997) 178.

[30] S. Kretzer, Phys. Rev. D 62 (2000) 054001.

[31] B.A. Kniehl, G. Kramer and B. Pötter, Phys. Rev. Lett. 85 (2000) 5288.

[32] S. Albino, B.A. Kniehl and G. Kramer, Nucl. Phys. B 725 (2005) 181, Nucl. Phys. B 803 (2008) 42.

[33] D. De Florian, R. Sassot and M. Stratmann, Phys. Rev. D 75 (2007) 114010, Phys. Rev. D 76 (2007) 074033.

[34] ZEUS Preliminary ICHEP2010, ZEUS-prel-10-013.

[35] G. Ingelman et al., Comput. Phys. Commun. 101 (1997) 108.

[36] H1 Preliminary DIS2010, H1 prelim-10-031. 